\title{
Charles Baudelaire, Arthur Rimbaud oder die Radikalität der Modernität
}

Wenn wir an dieser Stelle unserer Vorlesung erneut den Atlantik queren und zum französischen Lyriker Charles Baudelaire gelangen, dann nicht etwa deshalb, weil eine hübsche Mulattin im (Liebes-)Leben des Franzosen eine nicht unbedeutende Rolle spielte. Und wir tun dies auch nicht aus dem Grunde, dass Baudelaire sich sehr für einen literarischen Autor aus der Neuen Welt einsetzte, dessen schriftstellerisches Euvre er in Europa bekannt machte: Edgar Allan Poe. Vielmehr kann eine Vorlesung über das 19. Jahrhundert nicht auf jenen Dichter verzichten, der wie wohl kein anderer die rapide Modernisierung des Lebens nicht nur in Frankreich zum Ausdruck brachte und neue dichterische Mittel ersann, der von ihm beobachteten Modernität lyrischen Atem einzuhauchen. Damit öffnete er zugleich die Türen für jene literarischen Entwicklungen, welche Hugo Friedrich einmal in einer keinesfalls strukturalistisch gemeinten Wendung als die Struktur der modernen Lyrik bezeichnete. ${ }^{1}$ Nicht umsonst lautete dort der erste der Dichtkunst Baudelaires gewidmete Satz: „Mit Baudelaire wurde die französische Lyrik zu einer europäischen Angelegenheit. “2

Wir hatten Charles Baudelaire in unserer Vorlesung bereits kennengelernt in jenen Aussagen, die er in einem Brief an den Pariser Verleger Desnoyers von Ende 1853 oder Anfang 1854 gemacht hatte. Ich möchte Ihnen gerne diese Verabschiedung eines romantischen Naturbegriffs in diesen Zeilen noch einmal in Erinnerung rufen; denn es ist lange her, dass wir uns diese Überlegungen in Zusammenhang mit unserer Beschäftigung mit Giacomo Leopardi einmal näher anschauten. Damals schrieb Baudelaire an seinen ,lieben Desnoyers‘:

\footnotetext{
Sie bitten mich für Ihren kleinen Band um Verse, um Verse über die Natur, nicht wahr? Über die Wälder, die großen Eichen, das grüne Gras, die Insekten - die Sonne wohl auch? Aber Sie wissen doch nur zu gut, dass ich unfähig bin, mich über die Vegetabilien zu erweichen und dass meine Seele gegen jene sonderbare neue Religion rebelliert, welche stets, so will mir scheinen, für jedes spirituelle Wesen ich weiß nicht was an shocking besitzt. Ich werde niemals glauben, dass die Seele der Götter in den Pflanzen wohnt, und selbst wenn sie in ihnen wohnte, würde ich mich nur mäßig darum kümmern, und ich würde die meine von weit höherem Preise ästimieren als jene der geheiligten Gemüse. Ja, ich habe sogar stets gedacht, dass es in der blühenden und verjüngten Natur etwas Unvorsichtiges und etwas Traurig-Quälendes gibt. / Da ich mich in der Unmöglichkeit sehe, Ihnen vollständig Genüge
}

1 Vgl. Friedrich, Hugo: Die Struktur der modernen Lyrik. Von Baudelaire bis zur Gegenwart. Hamburg: Rowohlt 1956.

2 Ebda., S. 35. 
zu tun, schicke ich Ihnen, strikt den Begrifflichkeiten des Programms folgend, zwei poetische Stücke, welche in etwa die Summe der Träumereien repräsentieren, von denen ich in den Stunden der Dämmerung heimgesucht werde. In der Tiefe der Wälder, unter Gewölben eingeschlossen, welche Sakristeien und Kathedralen gleichen, denke ich an unsere erstaunlichen Städte, und die wundersame Musik, welche über die Gipfel rollt, erscheint mir wie die Übersetzung allen menschlichen Wehklagens. ${ }^{3}$

Innerhalb des langen Prozesses der Ablösung von den Formen und Normen der lange beherrschenden Romantik stellt diese Wendung gegen die „heiligen Gemüse" sicherlich eine der dezidiertesten Stellungnahmen dar, in denen ein Dichter seine Suche nach anderen Ausdrucksformen jenseits romantischer Naturlyrik kundtat. Es mögen solche Sätze Charles Baudelaires gewesen sein, die einen Literatur- und Kulturtheoretiker wie den jungen Roland Barthes dazu brachten, die Moderne nicht so sehr am Ausgang des 18. Jahrhunderts, sondern in der Mitte des 19. Jahrhunderts anzusetzen und beginnen zu lassen. Doch jenseits von Barthes' sehr unterschiedlichen und widersprüchlichen Modernebestimmungen stoßen wir bei Baudelaire auf eine neue, bisweilen geradezu revolutionäre Sichtweise der sich modernisierenden Großstädte, unter denen Paris - die Benjamin’sche „Hauptstadt des 19. Jahrhunderts“ - für ihn zweifellos das überragende Beispiel darstellte.

Mit großer ästhetischer Entschlossenheit und Vehemenz schlägt der am 9. April 1821 in Paris geborene und am 31. August 1867 ebendort verstorbene Charles Baudelaire die Tür zum Romantikverständnis Alphonse de Lamartines zu. Überhaupt schafft sich in seinen Gedichten, seinen Ausdruckswelten das Ästhetische radikal einen eigenen Raum, der sich von jener Transzendenz verabschiedet, die in allen Schöpfungen eines Lamartine noch angeklungen war und seit Chateaubriand zum unverzichtbaren Kernbestand romantischen Schreibens gehört hatte. Baudelaire verfügt über die gesamte Klaviatur christlicher Symbolik und Lehre; aber er glaubt nicht mehr an sie. Vielmehr räumt er mit christlichen Glaubenssätzen und ,Reliquien', aber auch mit Chateaubriands ästhetischem Christentum radikal auf und stellt den Menschen - vorzüglich in der Form des lyrischen Subjekts und gerne in der Inszenierung eines „Poète maudit“ - nun als Schöpfer, als „Créateur“ in den Mittelpunkt eines von Erschütterungen und Umwälzungen geprägten Geschehens. Dieses vermag ein mit hoher Sensibilität ausgestattetes, aber zugleich entpersönlichtes Subjekt in all seinen „tremblements“ seismologisch wiederzugeben. Jenseits jeglicher religiösen Transzendenz geht es um die hochsensible Erfassung all

3 Baudelaire, Charles: Lettre à Desnoyers (1853/1854). In (Ders.): Hommage à C. F. Denecourt. Fontainebleau : paysages - légendes - souvenirs - fantaisies. Texte établi par (préface de Auguste Luchet). Paris: Librairie de L. Hachette et Cie 1855, S. $73 \mathrm{f}$. 
jener Veränderungen, welche das zeitgenössische moderne Leben in Frankreich und im westlichen Europa ästhetisch prägen.

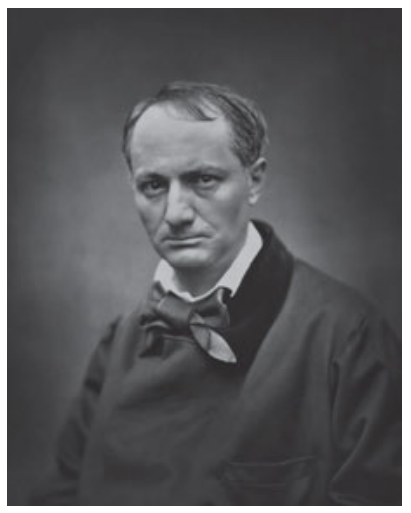

Abb. 67: Charles Baudelaire (Paris, 1821 - ebenda, 1867).

Die zweite Hälfte des 19. Jahrhunderts war in Frankreich und vielen Ländern Westeuropas ein Zeitraum starker sozioökonomischer Modernisierung, den adäquat zu erfassen die europäischen Großstädte der richtige Ort waren. Es fällt nicht leicht, diese sehr unterschiedlich ausgeprägten Entwicklungen übergreifend zu beschreiben, doch ist eine solch generelle Deskription von großer Wichtigkeit für eine Vorlesung, in welcher wir keineswegs nur die Modernisierungsprozesse in Frankreich erfassen, sondern auch Lateinamerika miteinbeziehen.

Arnold Hauser hat in seiner Sozialgeschichte der Kunst und Literatur, die erstmals nach dem Zweiten Weltkrieg im Jahr 1951 in einer ersten Ausgabe erschien, eine Art Kurzporträt dieser Zeit in groben, bisweilen holzschnittartigen Zügen gegeben. Noch in meiner Studentenzeit war dieser Band ein wichtiges Vademecum jedwedes Studierenden der Geisteswissenschaften, der sich für die Bezüge zwischen Literatur und Kunst einerseits und der Gesellschaft andererseits interessierte. Lesen wir dort den ersten Teil des nachfolgenden Zitats, so springen uns förmlich die Ähnlichkeiten zu uns viel näher liegenden Epochen ins Auge. Denn die Fortschritte von Technik und Technologie sind auch in unseren digitalisierten Zeiten keineswegs ruhiger geworden. Die Auswirkungen auf die Künste wie die Literaturen sind nicht weniger grundlegend, denn bestimmte pathologische Züge erkennen wir auch nach dem Ende der vierten Phase beschleunigter Globalisierung sofort - zumal dann, wenn wir noch die Auswirkungen der sogenannten Corona-Krise mitbedenken, die gesellschaftlich wie kulturell mit Sicherheit in vielfältiger Hinsicht einen Wendepunkt markieren.

Nach der zurückliegenden Beschleunigungsphase ist im Panorama weltgeschichtlicher Ereignisse eine zumindest in den westlichen Ländern leicht konstatierbare Krisenstimmung eingekehrt, die viele Bereiche des gesellschaftlichen Lebens 
erfasst hat und die Zukunftsaussichten unserer Demokratien durch die Gefahren eines immer gleichen, aber zyklisch wiederkehrenden Populismus bedroht. Ganz wie die Spanische Grippe am Ausgang der dritten Phase beschleunigter Globalisierung ruft Covid-19 eine allgemeine Desorientierung innerhalb einer globalgeschichtlichen und weltpolitischen Lage hervor, in welcher die Reichen immer reicher und die Armen immer ärmer werden. Dass sich diese Situation unter den noch immer vorherrschenden neoliberal-kapitalistischen Strukturen nicht mehr lange fortsetzen lässt, ist bei emotionsloser Betrachtung unter Einbeziehung der Klimakrise und der Verschlechterung menschheitlicher Lebensbedingungen sonnenklar. Und auch, dass in einer solchen hochkomplexen Situation die Populismen mit ihren einfachen, simplistischen Antworten blühen, überrascht in keiner Weise.

Wir sind vor dem Hintergrund dieser unserer zeitgenössischen Erfahrungen folglich bestens sensibilisiert für ein Verständnis all jener Veränderungen, die sich insbesondere im künstlerischen und literarischen Bereich unter dem Druck einer allseits ab Mitte des 19. Jahrhunderts herrschenden Modernisierungs- und Umwälzungsbewegung ergeben. So möchte ich Ihnen gerne Arnold Hausers Zitat anheimstellen. Ich tue dies nicht, weil dieser deutsch-ungarische Kunsthistoriker und Kunstsoziologe für meine eigene Studentengeneration so wichtige Impulse gab und überdies durch das kleine Biographem beeindruckte, im hohen Alter noch mit einigem Erfolg Chinesisch erlernt zu haben, sondern weil uns die nachfolgende Passage kurzgefasst einen Einblick in eine Zeit verschafft, deren Lyrik und Literatur wir so besser erfassen und verstehen können:

Die gewaltigen Fortschritte der Technik dürfen über die innere Krisenhaftigkeit der Zeitstimmung nicht hinwegtäuschen. Man muß vielmehr in der Krise selbst eine Anregung zu den technischen Errungenschaften und den Verbesserungen der Produktionsmethoden erblicken. Gewisse Züge der Krisenstimmung machen sich in allen Erscheinungsformen dieser Technik fühlbar. Es ist vor allem das rasende Tempo der Entwicklung und die Forciertheit der Veränderungen, die einen pathologischen Eindruck machen, besonders wenn man sie mit dem Gang der älteren Kulturgeschichte vergleicht, und ihre Auswirkungen in der Kunst vergleicht. Die rapide Entwicklung der Technik beschleunigt nämlich nicht nur den Wechsel der Moden, sondern auch die Verschiebung der künstlerischen Geschmackskriterien [...]. Mit dem Fortschritt der Technik verbindet sich als auffallendstes Phänomen die Entwicklung der Kulturzentren zu Großstädten im heutigen Sinne; diese bilden den Boden, in dem die neue Kunst wurzelt. Der Impressionismus ist eine par excellence städtische Kunst, und zwar nicht nur weil er die Stadt als Landschaft entdeckt und die Malerei vom Lande in die Stadt zurückbringt, sondern auch weil er die Welt mit den Augen des Städters sieht und auf die Eindrücke von außen mit den überspannten Nerven des modernen technischen Menschen reagiert. ${ }^{4}$

4 Hauser, Arnold: Sozialgeschichte der Kunst u. Literatur. München: Verlag C.H. Beck 1990, S. 928 f. 
Aus dieser kunstsoziologischen Perspektive erweist sich das lyrische Schaffen Charles Baudelaires geradezu als eine Anbahnung, eine Wegbereitung für jene künstlerischen Ausdrucksformen, die wie etwa der Impressionismus Antworten auf die Entwicklungen einer großstädtischen Zivilisation formulierten. Baudelaire fand ohne jeden Zweifel künstlerisch-literarische Lösungen für all jene Probleme, die er zu erleben, $\mathrm{zu}$ leben und auf herausragende Weise $\mathrm{zu}$ analysieren in der Lage war.

In einem seiner Tableaux parisiens der Fleurs du Mal griff Baudelaire eine typische Großstadtszene auf; eine Zufallsbegegnung, wie wir sie bereits bei Honoré de Balzac gleich zu Beginn von La Peau de Chagrin kennengelernt hatten. Ohne auf den Zufall - laut Balzac ja der „plus grand romancier du monde“ - noch einmal zurückzukommen, entfaltet der französische Dichter aus der Begegnung mit einer unbekannten Passantin auf der Straße eines seiner sicherlich schönsten Sonette:

\author{
La rue assourdissante autour de moi hurlait. \\ Longue, mince, en grand deuil, douleur majestueuse, \\ Une femme passe, d'une main fastueuse \\ Soulevant, balançant le feston et l'ourlet; \\ Agile et noble, avec sa jambe de statue. \\ Moi, je buvais, crispé comme un extravagant, \\ Dans son œil, ciel livide où germe l'ouragan, \\ La douceur qui fascine et le plaisir qui tue. \\ Un éclair ... puis la nuit! - Fugitive beauté \\ Dont le regard m'a fait soudainement renaître, \\ Ne te verrai-je plus que dans l'éternité? \\ Ailleurs, bien loin d'ici! trop tard! jamais peut-être! \\ Car j'ignore où tu fuis, tu ne sais où je vais, \\ Ô toi que j'eusse aimée, ô toi qui le savais! ${ }^{5}$
}

\title{
An eine, die vorüberging
}

Betäubend heulte die Straße rings um mich. Hochgewachsen schlank, in tiefer Trauer, hoheitsvoller Schmerz, ging eine Frau vorüber; üppig hob und wiegte ihre Hand des Kleides wellenhaften Saum;

5 Baudelaire, Charles: Tableaux parisiens, „A une passante“. In: Baudelaire, Charles: Die Blumen des Bösen / Les Fleurs du Mal. Vollständige zweisprachige Ausgabe. Aus dem Französischen übertragen, herausgegeben und kommentiert von Friedhelm Kemp. München: dtv 2002, S. 198 f. 
Leicht und edel setzte sie wie eine Statue das Bein. Ich aber trank, im Krampf wie ein Verzückter, aus ihrem Auge, einem fahlen, unwetterschwangeren Himmel, die Süße, die betört, die Lust, die tötet.

Ein Blitz ... und dann die Nacht! - Flüchtige Schönheit, von deren Blick ich plötzlich neu geboren war, soll ich dich in der Ewigkeit erst wiedersehen?

Anderswo, sehr weit von hier! zu spät! Niemals vielleicht! Denn ich weiß nicht, wohin du enteilst, du kennst den Weg nicht, den ich gehe, o du, die ich geliebt hätte, o du, die es wußte!

Das ursprünglich in der Zeitschrift L’Artiste im Jahr 1855 erstmals veröffentlichte und später in die zweite Auflage von Baudelaires Les Fleurs du Mal 1861 aufgenommene Gedicht folgt - so scheint es anfangs - der klassischen Form des Sonetts. Zugleich führt es aber durch eine Reihe von Enjambements, Einschnitten und Unterbrechungen, welche auch durch die Interpunktion angedeutet werden, auf der formalen Ebene eine fundamentale Unruhe ein. Diese Unruhe zeichnet geradezu seismographisch der erste Vers mit dem lauten Lärm der Straße, der auf das Ich einprasselt, sowie die extreme Spannung dieses lyrischen Ich auf, das zu einem zufälligen Beobachter einer eleganten, in Trauer gekleideten Frau wird, die auf der Straße vorübergeht. Es handelt sich um das Motiv der Zufallsbegegnung, ${ }^{6}$ der Begegnung, die im Grunde keine Begegnung ist.

So erscheint die Form des Sonetts nur auf den ersten Blick als klassisch, verkörpert sie doch die großstädtische Straßenszenerie mit ihrer ungebundenen Vektorizität, durch die sich zwei Figuren - ein Mann und eine Frau - in dieser Straßenszene für einen flüchtigen Augenblick als Menschen wahr-nehmen, sich vielleicht sogar als mögliche Liebespartner erkennen, sich aber dann wieder aus den Augen verlieren. Denn ihre Wege haben sich nur kurz gekreuzt. Die Parallele zu Balzacs Eingangsszenerie ist evident: War Balzacs männlicher Protagonist in La Peau de Chagrin durch diese Zufallsbegegnung wieder zum Leben zurückgekehrt, insofern er seine Selbstmordpläne auf Grund eines anmutigen Frauenknöchels fallen ließ, so fühlt sich auch das lyrische Ich beim Anblick der reizvollen Gestalt wie wiedergeboren, sieht sich „renaître“: Die schöne Unbekannte weckt im männlichen Ich alle Lebensgeister.

Vom ersten Vers an ist die Klanglandschaft, der Soundscape einer Großstadt, ja einer Metropole allgegenwärtig. Doch in diese Szenerie, die mit ihrem Lärm

6 Vgl. Leroy, Claude: Le mythe de la Passante de Baudelaire à Mandiargues. Paris: PUF 1999. 
nicht etwa als störend abgewertet wird, tritt eine lange, schlanke Frauengestalt, die im zweiten Quatrain des Sonetts mit allen Attributen einer bildhauerischen Schönheit ausgestattet wird. Erneut ist der Pygmalion-Mythos nicht weit entfernt, tritt aber zurück in eine Ausstattung der Dame, die in ihrem Auge gleichsam das Auge eines Hurrikans beherbergt.

Diese auf den ersten Blick überraschende Exotisierung der Unbekannten erfolgt keineswegs zufällig, findet sich in den Fleurs du Mal doch eine Vielzahl an Verweisen auf eine außereuropäische Welt, die oftmals im Indischen Ozean oder in der Karibik angesiedelt ist. So träumt sich das lyrische Ich in A une dame créole gleich im ersten Vers „Ins duftende Land, das die Sonne liebkost“, ${ }^{7}$ wo dieses Ich eine „dame créole“ - und „créole“ besitzt im Französischen eine andere Bedeutung als „criollo“ im Spanischen! - kennengelernt zu haben vorgibt. Zweifellos fließen in diese Darstellungen Erlebnisse einer 1841 durchgeführten Schiffsreise Baudelaires nach Mauritius und La Réunion ein, die in seinen Gedichten häufig am Horizont erscheinen. Diese schöne Dame würde - so heißt es im abschließenden Terzett dieses Sonetts - „tausend Sonette im Herzen der Dichter“ inspirieren, die von ihren Augen fasziniert wären: „Que vos grands yeux rendraient plus soumis que vos noirs." 8

Eine koloniale Welt erscheint unter der Feder des französischen Dichters, in welcher die sengende Sonne die exotischsten und extravagantesten Landschaften bescheint und wo Menschen leben, welche wie die hier erwähnten Schwarzen sich unter den Blick der (hier als weiblich apostrophierten) Herrschaft unterwerfen. Die in den französischen Überseegebieten zum damaligen Zeitpunkt abgeschaffte Sklaverei schimmert in ihrer metaphorischen Dimension als Sklaverei der Liebesbeziehung durch.

So zieht sich eine immer wieder aufblitzende, gleichsam kolonial-exotische Isotopie durch die Fleurs du Mal, die in den Tropen angesiedelt ist - bisweilen in der Karibik, bisweilen in Afrika, bisweilen in Indien. Das französische Kolonialreich erscheint in seiner weltweiten Ausdehnung als Ort der Träume und einer ganz anderen Gesellschaft, aber auch der erotischen Verlockungen, die von schwarzen, mulattischen, in jedem Falle nicht-weißen Frauenkörpern ausgehen. Beispielsweise heißt es gleich im ersten Vers des Gedichts A une Malabaraise: „Tes pieds sont aussi fins que tes mains et ta hanche / Est large à faire envie à la

7 Baudelaire, Charles: Les fleurs du mal. Edition établie selon un ordre nouveau, présentée et annotée par Yves Florenne. Préface de Marie-Jeanne Durry. Paris: Librairie Générale Française 1972, S. 83: „Au pays parfumé que le soleil caresse.“

8 Ebda., S. 84. 
plus belle blanche.“9 Die Erotisierung der Frau geht in den Fleurs du Mal mit ihrer Exotisierung einher; die Blumen des Bösen umweht ein dunkler, erotisierender Duft nach Tropen.

Spielen in allen erwähnten Gedichten die Augen der Schönen eine entscheidende Rolle, so verwundert es nicht mehr, wenn die Unbekannte Schönheit in $A$ une passante ebenfalls über das Auge des Hurrikans exotisiert und damit erotisiert wird. Denn selbstverständlich ist Paris nicht allein eine turbulente und laute Hauptstadt, sondern zugleich die Metropole eines französischen Kolonialreichs, das im 19. Jahrhundert zwar hinter dem britischen Empire zurückstand, gleichwohl aber ein koloniales Weltreich in seiner ganzen kulturellen Diversität darstellte. Mit anderen Worten: Paris konnte für Baudelaire sehr wohl zu einem WeltFraktal werden, ${ }^{10}$ in dem sich eine ganze Welt verkörperte und in welchem auch die Frauen in ihrer erotischen Exotik für das lyrische Ich allgegenwärtig waren.

Der Übergang in das vertrauliche Du konstruiert eine Nähe zu der Unbekannten, welche eine kurzzeitige räumliche Proximität - in der Kreuzung der beiden Wege - und eine kognitive, fast intime, wenn auch nur Sekunden andauernde Nähe herstellt: Denn das Du spürte nicht nur, sondern wusste von der Liebe des Ich. Doch der „coup de foudre“ wird allein vom dichter ausgestaltet und vom Dichter in all seinen Facetten gelebt. Denn Leben und Erleben kann man nicht nur eine gegebene Realität, sondern gerade auch die Fiktion, das Erfundene das, was uns wie in den Fleurs du Mal mit großer Obsession begleitet.

Wie ein Blitz durchzuckt die in Trauer gekleidete Unbekannte das Leben des Dichters, der danach wieder einsam in die Nacht zurückfällt. Die Isotopie des Exotischen und Fernen wird zu Beginn des zweiten Terzetts im „bien loin d'ici“" wieder aufgenommen, das zugleich einen intratextuellen und kotextuellen Hinweis auf ein gleichnamiges Gedicht in den Fleurs du Mal darstellt. Diese kotextuellen Netzwerke der Gedichte machen die besondere Faszinationskraft dieses herausragenden Gedichtbandes aus.

Doch nicht nur die Entrückung im Raum, sondern auch jene in der Zeit, die Entrückung in eine Ewigkeit und in ein Niemals, beschäftigen das Ich, das im „peut-être“ freilich noch eine letzte Hoffnung hegt. Der Leser, so Baudelaires Wendung an ihn gleich zur Eröffnung des Gedichtbandes, schaut dieser inneren Bilderflut, aber auch den sich anschließenden Fiktionen gebannt zu, ist er doch in Baudelaires Worten der „Hypocrite lecteur, - mon semblable, - mon frère!“11

9 Ebda., S. 254.

10 Zum Begriff des WeltFraktals vgl. Ette, Ottmar: WeltFraktale. Wege durch die Literaturen der Welt. Stuttgart: J.B. Metzler Verlag 2017.

11 Baudelaire, Charles: Les fleurs du mal, S. 7. 
Die Nacht birgt nicht länger wie in der Romantik die beiden Liebenden, sondern verhüllt die Unbekannte, entfernt die „Passante“, ohne dass es jenseits dieser Bewegung ins Finstere noch mehr gibt als ein Gedenken an jenen zurückliegenden, vergangenen Augenblick, in welchem sich eine ganze Welt, ein ganzes Leben mit all seinen Möglichkeiten eröffnete - und wieder schloss.

Eben diese Vektorizität und folglich dieselben Bewegungsrichtungen finden wir in einem Gedicht, welches auf das Jahr 1866 datiert ist und der Sammlung Les Epaves zugehört, die später Eingang in die Fleurs fanden. Diesmal aber gilt die Bewegung hin zur Nacht nicht einer schönen Frau, sondern einer uns nicht unbekannten Ästhetik, auf welche das folgende Sonett unter dem Titel Le Coucher du Soleil Romantique einen Abgesang darstellt:

\footnotetext{
Que le soleil est beau quand tout frais il se lève, Comme une explosion nous lançant son bonjour!

- bienheureux celui-là qui peut avec amour

Saluer son coucher plus glorieux qu'un rêve!

Je me souviens! ... J'ai vu tout, fleur, source, sillon, Se pâmer sous son œil comme un cœur qui palpite ...

- Courons vers l'horizon, il est tard, courons vite,

Pour attraper au moins un oblique rayon!

Mais je poursuis en vain le Dieu qui se retire; L'irrésistible Nuit établit son empire, Noire, humide, funeste et pleine de frissons;

Une odeur de tombeau dans les ténèbres nage, Et mon pied peureux froisse, au bord du marécage, Des crapauds imprévus et de froids limaçons. ${ }^{12}$
}

\section{Der Untergang der romantischen Sonne}

Wie schön die Sonne ist, wenn sie ganz frisch sich hebt und wie in einem Bersten ihren Morgengruß uns zuwirft! - Glückselig, wer in Liebe sie grüßen kann, wenn sie glorreicher als ein Traum im Glanze sinkt!

12 Baudelaire, Charles: Le Coucher du Soleil Romantique. In : Baudelaire, Charles : Die Blumen des Bösen / Les Fleurs du Mal, S. $296 \mathrm{f}$. 
Ich erinnere mich! ... Blume, Quelle, Furche, alles sah ich unter ihrem Auge sich regen wie ein schlagendes Herz ...- Laßt uns zum Rand der Erde laufen, es ist spät, rasch, laßt uns eilen, um wenigstens noch einen schrägen Strahl zu erhaschen!

Doch umsonst verfolge ich den Gott, der uns entweicht; unwiderstehlich breitet die Nacht ihre Herrschaft aus, schwarz, feucht, unheimlich und schaudervoll;

Ein Grashalm schwimmt in den Finsternissen, und unversehens tritt mein scheuer Fuß am Rand des Sumpfes auf Kröten und auf kalte Schnekcen.

Vektorizität durchzieht alle Quartette und Terzette dieses Sonetts, das lichtvoll zunächst mit einem Sonnenaufgang beginnt, durch dessen Schönheit in der Beschreibung freilich schon ein ironischer Schatten huscht: Denn alles scheint behaglich romantisch möbliert. Doch im Sonnenuntergang, dem romantischen Topos par excellence, steigt am Ausgang des ersten Quartetts etwas von dem Schatten bereits deutlich herauf, welcher mit der Herrschaft der Nacht die Terzette dominiert. Das dichterische Ich denkt zu Beginn des zweiten Quatrains an jene Zeit zurück, in der ihm die verschiedenen Elemente dieser (romantischen) Sprache noch geläufig waren. War Baudelaire in seinen frühen Jahren nicht mit dieser Ästhetik aufgewachsen? All diese einzelnen Elemente, die einen freundlichen Locus amoenus gestalten, in welchem ein Bächlein entspringt und wo die Blümelein sprießen, sind dem Ich vertraut und doch einer Vergangenheit überantwortet, an welche sich der Dichter nur zu gut zu erinnern vermag.

Doch diese Zeit ist vorüber: Es nützt nichts mehr, sich zu beeilen, schnell zu laufen, um noch das romantische Bild zu erhaschen, das ein Sonnenuntergang vielleicht bieten könnte - Es ist zu spät! Denn die Sonne geht unter und der Gott, der vom Génie du christianisme Chateaubriands getragene Gott, zieht sich zurück aus der Welt, die nun leer ist - Gott ist aus der Welt verschwunden. Und diese Welt wie ihr Dichter bleiben allein der Nacht überlassen, deren Majuskel ihre Sonderstellung hervorhebt. Das Reich der „Nuit“ hat längst begonnen, in welchem (um den Ausspruch Karls des Fünften und des Ersten von Spanien zu parodieren) die Sonne - zumindest die romantische Sonne - nicht mehr aufzugehen vermag. Wie im Gedicht $A$ une passante ist das Licht verloschen und eine Nacht hereingebrochen, die mit ihrer Finsternis alles „irrésistiblement“ einhüllt; nicht die romantische Nacht, die Zeit der Liebe und des Erzählens, sondern jene unwiderstehliche Nacht, die Tod und Fäulnis herbeiruft.

So wird im letzten Terzett die Sumpflandschaft auch von einem Geruch nach Tod durchdrungen; eine „odeur de tombeau“ liegt über einer Landschaft, in 
welcher der Fuß des Ich den Gesichtssinn ersetzt und im Finstern sich angstvoll vorantastend zum Sehorgan wird. Die Finsternisse gemahnen an die parallele Vektorizität im Gedicht an die Unbekannte, wo alles in einer Unendlichkeit, einer leeren Ewigkeit zu vergehen droht, ohne noch einmal vom Blitz, vom „coup de foudre“ erhellt zu werden. Diese gefährliche und glitschige Ewigkeit steht am Ende, nach dem Sonnenuntergang der Romantik, als Ausgang aus einer Epoche, deren längst vergangenes, untergegangenes Licht das Gedicht nicht leugnet und nicht verklärt.

Bei Baudelaire erscheint kein Jubel, kein Lichtschein am Horizont, keine versöhnende Vision, sondern der Bruch mit dem Alten, das nicht mehr gelten kann. Das Ich des Dichters macht auf ein entpersönlichtes Subjekt aufmerksam, welches diesen einen Menschen, dieses dichterische Ich, zum Brennpunkt eines wenn nötig die ganze Menschheit umfassenden Denkens macht und aus einem solchen nur scheinbar individuellen Prisma den ganzen Glanz seiner verdichteten Bilder bezieht. Es ist gewiss nicht falsch, in diesem Ich mit Hugo Friedrich den „Erleider der Modernität“13 zu sehen. Doch haftet Friedrichs Buch vielleicht zu viel Exorzismus, zu viel Austreibung des für den Freiburger Romanisten in die Lyrik gekommenen Teufels an, als dass wir Baudelaire allein in dieser Rolle sehen dürfen. Denn war nicht er es, der forderte, dass es heutzutage gelte ,absolut modern“ zu sein? Und wurde nicht Baudelaire zum Wegbereiter einer Radikalität, die wir sogleich in der Lyrik Arthur Rimbauds aufscheinen sehen werden, zum Wegbereiter einer Dichtkunst, die unbezweifelbar direkt ins 20. Jahrhundert führen sollte?

Es ist an dieser Stelle unserer Vorlesung nicht möglich, Les Fleurs du Mal in ihrer Gesamtheit zu portraitieren und als Gesamtheit zu verstehen: in ihrer Gesamt-Komposition von einem Anfang bis an ein Ende, in ihrem intratextuellen und kotextuellen Verwoben-Sein, in welchem jedes Gedicht für sich allein und wie eine Insel zugleich mit allen andern Inseln in Kontakt und Austausch steht. Durch diese strenge Formgebung verstand es Baudelaire, seinen einzelnen, aus unterschiedlichen Kontexten entstandenen Gedichten etwas vom konkreten Raum Befreites und nahezu Überzeitliches zu geben. Dabei bemühte sich der französische Dichter, seinen Blumen des Bösen nicht zu viel Wohlgeruch und zu viel Schönheit mitzugeben, um geradezu obsessiv die ihn bedrückende Nachtseite zu schildern, die nicht mehr die Nachtseite der Vernunft, sondern die der Existenz war. Dabei ist es faszinierend zu sehen, mit welchen schriftstellerischen und dichterischen Mitteln es ihm gelingt - ähnlich wie ein ebenfalls gegen die

13 Friedrich, mHugo: Die Struktur der moderenen Lyrik, S. 37. 
Romantik anschreibender Gustave Flaubert ${ }^{14}$ - gegen alle Immoralismus-Vorwürfe seiner Zeit ${ }^{15}$ ein zutiefst moralisches Buch zur Unmoral zu schaffen und dabei jene weltumspannende Dimension einzubauen, in welcher die französische Modernität zur Grundstruktur eines WeltFraktals gerät.

Als (zeitweise vom ererbten Geld finanziell abgesicherter) Dandy und Bohemien, als Fourier'scher Sozialist und gescheiterter Anhänger der Februarrevolution von 1848, als mit der Romantik verwobener und doch radikal aus ihr ausbrechender Dichter, aber auch als Übersetzer Edgar Allan Poes, als Kunst- und Literaturkritiker schritt Baudelaire bis zu seinem Schlaganfall und Tod die verschiedensten Facetten der Moderne ab. Als „Peintre de la vie moderne“ lebte er mit größter Konsequenz die unterschiedlichsten Aspekte des modernen Lebens, das er in seiner vielleicht letzten Rolle als erster der großen „poètes maudits“ mit allen Widersprüchen wohl am überzeugendsten verkörpern konnte. Baudelaires Modernität zeichnet sich durch seinen unbeugsamen Willen zur ästhetischen Durchdringung aus, ja durch eine Radikalität, die seine Dichtung zum einen zur Grundlage aller künftigen lyrischen Entwicklung, zum anderen aber ihn selbst zum Vorläufer und vielleicht mehr noch Wegbereiter eines anderen Dichtergenies werden ließ. Dieses sollte nur für kurze Zeit im französischen Parnasse aufblitzen, und mit ihm werden wir uns im weiteren Verlauf dieses Kapitels beschäftigen.

Jean Nicolas Arthur Rimbaud wurde am 20. Oktober 1854 im französischen Charleville geboren und starb am 10. November 1891 in Marseille. ${ }^{16}$ Als hochbegabtes Kind war er der ganze Stolz seiner streng religiösen Mutter und brillierte schon früh, im Alter von sechs oder sieben Jahren, mit allerlei Gelegenheitsgedichten, die auch in Lehrerzeitschriften abgedruckt wurden. Dass er schon bald aus der provinziellen Enge seiner Welt, aber auch aus seiner strengen Erziehung durch die Mutter ausbrechen sollte, war geradezu vorprogrammiert. Das von ihm wie von seiner Mutter angestrebte Abitur sollte der junge Mann niemals erreichen.

Während des Preußisch-Französischen Krieges lag Rimbauds Geburtsort nahe der Frontlinie und so nutzte dieser das allgemeine Tohuwabohu, um erstmals nach Paris zu gelangen - der auch von ihm erträumten Metropole. Er sym-

14 Vgl. den Gustave Flaubert gewidmeten Teil im zweiten Band der Reihe „Aula“ in Ette, Ottmar: LiebeLesen, S. 677-683.

15 Vgl. hierzu Heitmann, Klaus: Der Immoralismusprozeß gegen die französische Literatur im 19. Jahrhundert. Bad Homburg - Berlin - Zürich: Verlag Gehlen 1970.

16 Zur Biographie vgl. Bonnefoy, Yves: Arthur Rimbaud in Selbstzeugnissen und Bilddokumenten. Reinbek bei Hamburg: Rowohlt 1980. 


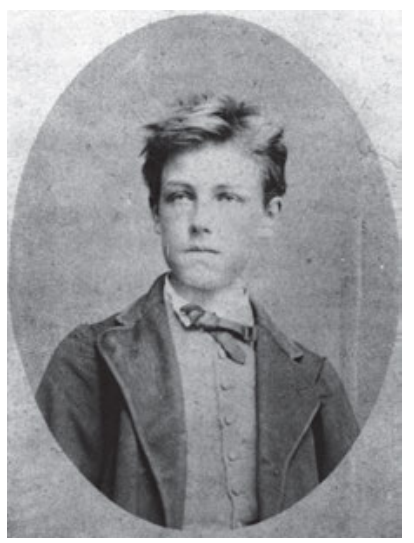

Abb. 68: Arthur Rimbaud (Charleville, Frankreich, 1854 - Marseille, 1891), circa 1871.

pathisierte mit der Kommune, mit dem Aufstand der Pariser Arbeiter, und erlebte zumindest am Rande die Schrecken des Krieges. Inwieweit er aktiv an Aktivitäten der „Communards“ teilnahm, ist bis heute umstritten, doch dürfte seine Teilnahme eher ideeller Natur gewesen sein. Dennoch zog es Rimbaud damals, wie später auch, stets mitten ins Geschehen. ${ }^{17}$

An die blutige Epoche der französisch-deutschen Beziehungen erinnert sein Gedicht Le Dormeur du Val, Der Schläfer im Tal, ein auf Oktober 1870 datiertes Sonett, an dem uns freilich nicht interessiert, ob Arthur Rimbaud diese Szenerie selbst miterlebt hat oder nicht. Meine Übersetzung lehnt sich an eine frühere, kühne Übertragung von Stefan George an:

\footnotetext{
Ein grünes Loch, das ein Bach befeuchtet Der toll das Gras mit Silberflecken säumt, Wohin vom stolzen Berg die Sonne leuchtet, Ein kleines Tal ist's, das von Strahlen schäumt.

Ein Soldat, jung, barhaupt, mit offnem Munde Den Nacken badend in dem blauen Kraut, Unter freiem Himmel schläft; bleich, am Grunde ins grüne Bett gestreckt, vom Licht betaut.

Sein Fuß in Gladiolen steckt. Und wie ein Kind, Das krank ist, lächelnd: Hält er seinen Schlummer. Natur, umhüll ihn warm: Es friert ihn noch.
}

17 Vgl. auch Wagner, Horst: Arthur Rimbaud. In: Lange, Wolf-Dieter (Hg.): Französische Literatur des 19. Jahrhunderts. Bd. III: Naturalismus und Symbolismus. Heidelberg: Quelle \& Meyer 1980, S. 101-120; sowie Murat, Michel: L’Art de Rimbaud. Paris: José Corti 2002. 
Ihm zuckt die Nase nicht vom duftigen Wind.

Er schläft im Sonnenschein, die Hand auf stummer

Brust. Auf rechts, verdoppelt, ist ein rotes Loch.

C'est un trou de verdure où chante une rivière Accrochant follement aux herbes des haillons d'Argent; où le soleil, de la montagne fière, Luit: c'est un petit val qui mousse de rayons.

Un soldat jeune, bouche ouverte, tête nue, Et la nuque baignant dans le frais cresson bleu, Dort; il est étendu dans l'herbe, sous la nue, Pâle dans son lit vert où la lumière pleut.

Les pieds dans les glaïeuls, il dort. Souriant comme Sourirait un enfant malade, il fait un somme: Nature, berce-le chaudement: il a froid.

Les parfums ne font pas frissonner sa narine; Il dort dans le soleil, la main sur la poitrine Tranquille. Il a deux trous rouges au côté droit. ${ }^{18}$

Dieses Sonett gehört wohl zu den berühmtesten Anti-Kriegsgedichten, die jemals geschrieben wurden. Dabei kommt es gar nicht wie ein solches daher: Arthur Rimbaud zögert nicht, in auf den ersten Blick klassischen Alexandrinern einen Locus amoenus nach antikem (frühromantischem) Vorbild zu entwerfen, um dieses schöne Bild mit Blümelein und Bächlein und allerlei grünem Kraut fast das ganze Sonett über in Spannung zu halten. Doch das „trou“, das Loch des grünen Winkels, weist bereits auf die beiden roten „trous“ im letzten und entscheidenden Vers des Gedichts voraus: Was sich als blühender Gemeinplatz und als Lob der direkt aufgerufenen Mutter Natur tarnt, ist nichts anderes als Dekor für einen tödlich verwundeten, im Kriege erschossenen und sinnlos gefallenen Soldaten.

Der gerade einmal sechzehnjährige Dichter ist, wie die kunstvollen Alexandriner zeigen, im Vollbesitz seiner lyrischen Kräfte. Doch er streut durch die zahlreichen Enjambements, die eingefügten Bruchlinien und die ein ruhiges, kontinuierlich zu genießendes Naturbild konterkarierende Syntax einen unruhigen, unsteten Rhythmus in die vertrauten Formen. Mit seinem ersten „trou“ setzt er

18 Rimbaud, Arthur: Le Dormeur du Val. In: ders.: Euvres. Sommaire biographique, Introduction, notices, relevé de variantes et notes par Suzanne Bernard. Edition illustrée. Paris: Edition Garnier Frères 1961, S. 76. 
bereits im ersten Vers mit einer erstaunlichen Wendung, die in Stefan Georges schöner Übertragung freilich fehlt, einen Spannungsbogen, der sich bis in den letzten Vers des zweiten Terzetts erstreckt. Denn die von Rimbaud entworfene Szenerie zeigt uns nicht einen Schläfer in idyllischer Umgebung, sondern den Schlaf eines Toten, der als Soldat im Preußisch-Französischen Krieg fiel - gleich auf welcher Seite! Wir haben es nicht mit einer Anklage gegen diese oder jene Kriegspartei zu tun, sondern mit einer Anklage gegen den Krieg selbst.

Rimbaud wirbelte in den wenigen Jahren seiner dichterischen Existenz die französische Lyrik kräftig durcheinander. Er ließ in seiner jugendlichen Radikalität außer Baudelaire, Verlaine und Hugo kaum einen Dichter gelten, zielte auf Effekte, die vor ihm selten so scharf gesehen worden waren und verband alles zu einer Synästhesie, die in ihren ausgelassenen Feiern Vokale und Farben, Rhythmen und Töne, Klangfarben und Schriftbilder miteinander lustvoll vermischte. So heißt es in seinem Gedicht Voyelles gleich im berühmten Eingangs-Vers zu den Vokalen: „A noir, E blanc, I rouge, U vert, O bleu: voyelles“ („A schwarz E weiß I rot U grün O blau - Vokale“). ${ }^{19}$ Dabei habe ich mich stets gefragt, ob das grüne U nicht Alfred Jarry zu seinem Ubu und La chandelle verte, seiner grünen Kerze inspiriert haben mag, mit welcher der lange Weg durch ein 20. Jahrhundert begann, in welchem die angstbesessenen und darum umso sadistischeren Tyrannen überall an die Macht kamen. ${ }^{20}$ Jarry hat sie alle, bis in unsere Tage, portraitiert.

Wollte man Rimbauds gesamte Existenz charakterisieren, so könnte man auf die Eingangsworte von Hugo Friedrichs Rimbaud-Kapitel zurückgreifen:

Ein Leben von siebenunddreißig Jahren; ein im Knabenalter beginnendes Dichten, das nach vier Jahren abbricht; der Rest, bei völligem literarischem Schweigen, ein unruhiges Umherreisen, das am liebsten bis nach Asien vorgedrungen wäre, sich aber mit dem Nahen Osten und Zentralafrika begnügen mußte, ausgefüllt von vielerlei Beschäftigungen in Kolonialarmeen, Steinbrüchen, Exportfirmen, schließlich im Waffenhandel für den Negus von Abessinien, und nebenbei von Berichten an geographische Gesellschaften über bisher unerforschte Gebiete Afrikas; innerhalb jener knappen Dichterzeit ein rasendes Tempo der Entwicklung, die schon nach zwei Jahren dazu geführt hatte, den eigenen Anfang, aber auch die hinter diesen stehende literarische Tradition zu sprengen und eine Sprache zu schaffen, die bis heute eine Ursprache moderner Lyrik geblieben ist: das sind einige Tatsachen der Person Rimbauds. ${ }^{21}$

19 Ebda., S. 110.

20 Die Vervielfachung der Figur des Ubu roi schmückt denn auch meinen Band mit den Vorlesungen zum 20. und 21. Jahrhundert in Ette, Ottmar: Von den historischen Avantgarden bis nach der Postmoderne (2021), Cover.

21 Friedrich, Hugo: Die Struktur der modernen Lyrik, S. 59. 
So lässt sich kurz gefasst resümieren, welche Urgewalten in diesem jungen Mann steckten, der gewiss Baudelaire stets in Ehren hielt und seine ästhetischen Doktrinen befolgte, aber noch in seinem aus heutiger Sicht spektakulären Verstummen ein Statement zur künftigen Entwicklung moderner Dichtkunst abgab.

Vielleicht geben uns am besten die beiden am 12. und 15. Mai 1871 verfassten Briefe eine Auskunft, die als Lettres du voyant, als die Briefe des Sehers in die Literaturgeschichte eingingen und denen in Deutschland Werner von Koppenfels eine schöne zweisprachige Ausgabe widmete. ${ }^{22}$ In diesen beiden Briefen an unterschiedliche Adressaten, die uns in diesem Zusammenhang nicht näher zu interessieren brauchen, formuliert Arthur Rimbaud jene Wendungen, die in keiner Darstellung moderner Lyrik, aber auch in keiner Theorie der Autobiographie bis heute fehlen dürfen und die uns Einblick in jegliche Fadenscheinigkeit des Identitätsbegriffs geben, wenn wir ihn auf die Literatur anzuwenden versuchen. In ihren Worten wird der Dichter zum Seher, der wie Gott in seinem ureigenen Universum herrscht:

I.

Ich ist ein Anderer. Was soll man machen, wenn das Holz auf einmal Violine wird? Ein Hohngelächter all den Ahnungslosen, die über Dinge räsonnieren, von denen sie nicht das Geringste verstehen!

Für mich sind Sie keine Lehrkraft. Ich schenke Ihnen dies hier: ist es Satire, wie Sie sagen würden? Ist es Poesie? Jedenfalls ist es eine Fantasie.

II.

Ich sage, man muß Seher sein, muß sich sehend machen.

Sehend macht sich der Dichter durch eine lange, unermeßliche und planmäßige Ausschweifung aller Sinne. Alle Formen der Liebe, der Qual, des Wahnsinns; er sucht eigens, er erschöpft an sich alle Gifte, um nur ihre Quintessenz zu bewahren. Unsägliche Tortur, für die er allen Glauben braucht, alle übermenschliche Kraft, bei der er unter allen der große Kranke wird, der große Verbrecher, der große Verdammte; und der höchste Wissende!

I.

Je est un autre. Tant pis pour le bois qui se trouve violon, et Nargue aux inconscients, qui ergotent sur ce qu'ils ignorent tout à fait!

Vous n'êtes pas Enseignant pour moi. Je vous donne ceci: est-ce que de la satire, comme vous diriez? Est-ce de la poésie? C’est de la fantaisie, toujours.

II.

Je dis qu'il faut être voyant, se faire voyant.

Le Poète se fait voyant par un long, immense et raisonné dérèglement de tous les sens. Toutes les formes d'amour, de souffrance, de folie; il cherche lui-même, il épuise en lui tous les

22 Rimbaud, Arthur: Seher-Briefe. Lettres du voyant. Übersetzt und herausgegeben von Werner von Koppenfels. Mainz: Dieterich’sche Verlagsbuchhandlung 1990. 
poisons, pour n'en garder que les quintessences. Ineffable torture où il a besoin de toute la foi, de toute la force surhumaine, où il devient entre tous le grand malade, le grand criminel, le grand maudit, - et le suprême Savant! ${ }^{23}$

In diesen kurzen Auszügen wird die absolute Art, die unerhörte Radikalität deutlich, mit der Rimbaud seinen früheren Begleiter und Berater, aber auch allgemein den Rest der Menschheit behandelt: Es ist ein absoluter Wille, zur Essenz der Dinge vorzustoßen und sich nicht mit dem zufrieden zu geben, was als gängig gelten mag. Das Ich des Dichters ist ein Anderer - und in diesem Anderssein tauchen viele Positionen eines Ich auf, wie man sie schon in Baudelaires Dichtungskunst mit ihren entpersönlichten lyrischen Ich-Figuren erkennen mag. Es ist nicht mehr das romantische Ich mit seiner Tiefe und seiner Zerrissenheit, sondern eine Figur, die ständig - so könnten wir ironisch sagen - ihre Identität wie ihre Hemden, Gedichte und Stellungen wechselt.

Gewiss schreibt sich vor allem im zweiten Seher-Brief die Aufwertung des Dichters in der Romantik zum „Créateur“ fort, zum Schöpfer und Demiurgen, so dass wir in diesem Zusammenhang durchaus Kontinuitäten erkennen können. Aber wie nahe sind wir hier doch dem Fin de siécle, wie nahe dem nietzscheanischen Übermenschen, wie nahe einer Künstler- und Dichterfigur, die alles zu erkennen vermag, was sich der Vernunft und dem Nachdenken der Mitmenschen entzieht! Zwar gibt es Kontinuitäten mit der Romantik; doch handelt es sich insgesamt um einen Bruch, der jenen gesuchten und gefundenen Bruchlinien gleicht, wie sie die historischen Avantgarden zu Beginn des 20. Jahrhunderts und insbesondere der französische Surrealismus zelebrierten. Es kann daher nicht verwundern, dass es gerade französische Surrealisten waren, die in Arthur Rimbaud einen der Ihren erkannten und sich seiner Dichtungskonzeption anschlossen.

Doch glauben wir nicht, dass es bei Arthur Rimbaud um ein dichterisches Sehen im Sinne einer profunden Irrationalität ginge. Vielmehr hebt der französische Dichter das vernunftgemäße, das vernunftbetonte „dérèglement de tous les sens" hervor; eine Dichtungslehre, welche die historischen Avantgarden und einen großen Teil der modernen europäischen Lyrik faszinierte. Denn die übermenschliche Anstrengung, von der Rimbaud spricht, ist nicht nur ein Heraustreten des Dichters aus allen gesellschaftlichen Bezügen, ist nicht nur ein Kriminell-Werden, das an den surrealistischen Gestus denken lässt. Es erinnert etwa an die Überlegung, auf die Straße hinunter zu gehen und einfach einen Passanten abzuknallen - ein Kriminell-Werden, das noch in Stockhausens Zelebrieren des Attentats auf das World Trade Center zu spüren ist in der ästhetischen Feier der

23 Rimbaud, Arthur: Seher-Briefe. Lettres du voyant, S. 10-12 und 24 f. 
Schönheit der einstürzenden Twin Towers. Es ist zugleich ein Wissen, das auch die Wissenschaft miteinschließt, sich aber nicht auf sie begrenzt; das alle Vernunft mobilisiert, aber nicht bei der Vernunft allein stehenbleibt. Wir erkennen in diesem Dichter etwas von jenen Figuren Friedrich Nietzsches, die in dem Tänzer Zarathustra die Choreographien der Vernunft, der Poesie und des Wahnsinns miteinander fusioniert und hochleben lassen.

Rimbauds „Entregelung aller Sinne“, wie wir es vielleicht besser übersetzen könnten - denn die „Ausschweifung“ in der obigen Übersetzung gibt nichts von der Metaphorik der bewussten Ent-Regulierung zum Ausdruck -, ruft das Kalkül auf, die kalte Vernunft, die sich des Wortmaterials, der Semantik, der Metrik, der Musikalität bemächtigt. Sie soll alles verstellen, alles entstellen, um mit den Traditionen zu brechen, an die der Dichter anzuknüpfen nicht umhin kam, die er aber ablehnt, da sie ihn nicht zu den Quintessenzen des Eigentlichen und Absoluten führen. Das ist ein völlig neuer Ton, das sind gänzlich neuartige Gesten einer Dichterfigur, die den Romantiker in sich längst erdrosselt hat, um zu radikal Neuem fähig zu sein und neue Horizonte auszumachen!

Die rationale Entregelung aller Sinne bedeutet nicht etwa, dass etwa der Alexandriner als royale Versform aufgegeben, sondern dass dieser Zwölfsilber durchlöchert und zerhackt wird, dass sein Wortmaterial zu einem Farbenmaterial, zu einem Musikmaterial transformiert werden muss, in welchem die von Rimbaud so sehr geschätzten Synästhesien in ein Gesamtkunstwerk einfließen, wie es zeitgleich einem Richard Wagner vor der Seele schwebte. An dieser Stelle wird auch deutlich, dass in eine solche Kunst-Landschaft keine feste Identität mehr zu passen vermag, dass die künstlerische Identität keine mehr eines selbstidentischen Ich, sondern die Identitätsfigurationen unterschiedlicher Stellungen und Positionen, unterschiedlicher Bewegungsfiguren und Choreographien ist, wie sie auch bei Nietzsche einem Tänzer namens Zarathustra zukommen.

Dass einer solchen ästhetischen, dichterischen Figuration mit all ihren synästhetischen Konsequenzen keine kontinuierliche Gesellschafts-Struktur mehr entsprechen kann, sondern dass der Bruch mit allen Traditionen auch einem Bruch mit dem herkömmlichen Gesellschaftssystem entsprechen muss, zeigte sich in gewisser Weise bereits im Gedicht Le Dormeur du val. Denn mit Hilfe dieses Schläfers im Tal wird der Krieg angeprangert, der von all jenen Mächtigen entfacht wurde, welche so sehr die wunderschönen Landschaften und Topoi der Romantik lieben; ein Krieg, den freilich all jene mit dem Leben bezahlen, die als einfache Soldaten zum ,Kanonenfutter‘ der gegeneinander gehetzten Armeen werden.

Binnen weniger Jahre war der brillante Schüler und Verfasser von systemstabilisierenden Gelegenheitsgedichten, in welchem man niemals den kommenden Rebellen vermutet hätte, zu jenem Sechzehnjährigen geworden, der nicht nur dieses Anti-Kriegsgedicht verfasste, sondern mit seinem Spott auf all 
das üblicherweise als ,schön' Geachtete bald schon vehement die Hässlichkeit pries und einer Venus körperliche Defizienzen andichtete. Die wilde, damals gesetzeswidrige Liebesbeziehung mit dem großen Dichter Paul Verlaine, der für die Rettung vieler Gedichte Rimbauds verantwortlich zeichnete und mit dem der junge Rimbaud umhervagabundierte, wurde zum Inbegriff eines Verlangens nach Liebe, die der junge französische Dichter mit zunehmender Verzweiflung suchte und nicht finden konnte. In den Lettres du voyant nimmt Rimbaud den Begriff des „maudit“ selbst in den Mund, weiß sich zudem als „criminel“, verfügt aber auch über ein Wissen, das dieses Wunderkind der französischen Literatur aus der Masse dichtender Zeitgenossen heraushebt.

Seit seinem ersten, jäh unterbrochenen Ausflug in die französische Hauptstadt hatte sich das Bild der Pariser Kommune dem Dichter des ästhetischen Bruchs als Sinnbild eines Aufstands gegen alles und einer Niederwerfung jeglicher Ordnung eingeprägt. Die erträumte Vereinigung mit den „Communards“, mit denen Rimbaud sympathisierte, zeigt sich in jener Dichtung eines entregelten Ich, das sicherlich ein Anderer und zugleich doch ein Rimbaud ist, mit dessen Namen das Gedicht signiert ist. Zugleich ist es eine Antwort auf jene Landschaftsszenerien, welche Lamartine unter dem Nordwind des „Aquilon“ entwarf:

Was ist uns, mein Herz, all diese Lachen von Blut

Und Glut, und tausenderlei Mord, und der Schreie Hall

Der ganzen Hölle alle Ordnung stürzend, und der Wut

Schrei; auch noch der Nordsturm, über Trümmern überall;

Und all die Rache? Nichts? ... Aber doch, noch immer rein, Wir wollen sie! Industrielle, Fürsten, Wissensmacht:

Zugrund mit Euch! Geschichte, Recht und Macht: Niedergemacht!

Gehört’s doch uns! Glut, Glut, der güldnen Flamme Schein!

Alles dem Krieg, der Rache, dem Terror sei,

Mein Geist! Bohrn wir das Messer in der Wunde! Ah, der Flug

Der Republiken dieser Welt, stürz ab! An Kai-

sern, Regimentern, Siedlern, Völkern ist’s für alle Mal genug!

Wer sonst heizt an den großen Wirbelsturm der Glut

Als wir und alle jene, die wir zu Brüdern lachen?

Her zu uns, Freunde ihr, das wird uns Freude machen.

Nie werden wir arbeiten, niemals! Herbei, des Feuers Flut!

Europa, Asien, Amerika, weh Euch, vergeht!

Unser Rächermarsch wird alle Ort besetzen,

Städte und Länder, wir werden hier zergehn!

Vulkane sollen platzen! Ozeane soll'n zerfetzen! ... 
Oh meine Freunde! Sag, Herz, denn sicher sind es Brüder:

Ihr unbekannten Schwarzen, gingen wir! Auf, gehn wir los!

Oh Unglück! Ich fühle mich erbeben, die alte Erde,

Sie packt mich, der ich immer mehr zu Euch! Ihr Schoß,

Und doch ist's nichts! Hier bin ich! Bin ich noch immer. ${ }^{24}$

Qu'est-ce pour nous, mon cœur, que les nappes de sang

Et de braise, et mille meurtres, et les longs cris

De rage, sanglots de tout enfer renversant

Tout ordre; et l'Aquilon encor sur les débris;

Et toute vengeance? Rien! ... Mais si, toute encor,

Nous la voulons! Industriels, princes, sénats:

Périssez! puissance, justice, histoire: à bas!

ça nous est dû. Le sang! le sang! la flamme d'or!

Tout à la guerre, à la vengeance, à la terreur, Mon esprit! Tournons la morsure: Ah! passez, Républiques de ce monde! Des empereurs, Des régiments, des colons, des peuples, assez!

Qui remuerait les tourbillons de feu furieux, Que nous et ceux que nous nous imaginons frères? A nous, romanesques amis: ça va nous plaire. Jamais nous ne travaillerons, ô flots de feu!

Europe, Asie, Amérique, disparaissez.

Notre marche vengeresse a tout occupé,

Cités et campagnes! - Nous serons écrasés!

Les volcans sauteront! Et l'Océan frappé ...

Oh! mes amis - Mon cœur, c'est sûr, ce sont des frères:

Noirs inconnus, si nous allions! Allons! allons!

O malheur! je me sens frémir, la vieille terre,

Sur moi de plus en plus à vous! la terre fond,

Ce n'est rien! j'y suis! j'y suis toujours.

Dieses aus dem Jahre 1872 stammende Gedicht zählt zweifellos zu den großen lyrischen Schöpfungen des Sehers und „Poète maudit“. Als lyrische Schöpfung

24 Rimbaud, Arthur: Qu'est-ce pour nous, mon cœur. In: ders.: Les Illuminations. Texte établi par Félix Fénéon, Paris: Publications de la Vogue 1886, S. 98-99. 
von ausschließlich männlichen Reimen greift Rimbaud auf Quartette zurück, die sich eines erkennbaren Zwölfsilbers erfreuen: Noch immer also dient ein Alexandriner als Grundlage des dichterischen Sehens, doch was ist aus ihm geworden! Er ist zerrissen, fast würde man sagen zerschossen, so durchsiebt ist er mit Brüchen, Lücken, Enjambements, Zäsuren und Rhythmuswechseln. Und doch sind es die Zwölfsilber, die allem ihre dichterische Form geben, bis auf den letzten Vers, der lediglich, je nach Zählweise, neun oder zehn Silben besitzt. An dieser Stelle dürfen wir sehr wohl die beiden Silben des Namens „Rimbaud“ ergänzen, der sein Gedicht gleichsam in absentia unten rechts signiert. Aber ist es der französische Dichter wirklich selbst und nicht viel mehr der Andere, die Anderen?

Wo wir bei Lamartine sich eine verlassene Landschaft vor dem Dichterauge auftun sehen, tritt uns bei Rimbaud von der ersten Zeile an das vergossene Blut, schlägt uns die fortbestehende Glut entgegen, die noch immer in den Trümmern lodert. Der Nordwind, ja der Nordsturm des „Aquilon“ ist ganz zweifellos das Vorrücken des preußischen Adlers, des „Aquila“, der sich des lodernden Paris bemächtigt. Gleichzeitig handelt es sich um ein Augenzwinkern an die Leserschaft, welche die romantische Diktion sofort versteht. Versübergreifend zwischen drittem und viertem Vers wird jegliche Ordnung umgestürzt. Es sind Bilder der Zerstörung, gewiss, aber auch Bilder der Hoffnung, bricht doch die bisherige Macht in sich zusammen: „Renversant Tout Ordre.“ Und diesem Inhalt entspricht überdeutlich auch die Form: Die Alexandriner sind noch erkennbar, doch wirken sie in ihrem gesamten Aufbau wie zerstört und durchlöchert. Poetische Vision und dichterische Form sind also geradezu klassisch aufs Engste miteinander verbunden, freilich auf der Grundlage einer Zerstörung, welche sowohl die poetischen Bilder als auch die lyrischen Ausdrucksmittel ergreift.

Doch was soll an die Stelle des Zerstörten treten? Allein die Rache, die das Ich sich schwört, kann nicht genügen. Die alte Herrschaft nicht nur im politischen, sondern auch im wirtschaftlichen Bereich wird aufgezählt, bevor sie der Dichter in den Abgrund wirft. Nieder mit allem! Aber das Blut, das dieses Ich vergießt und anruft, reicht nicht aus. Eine neue Brüderlichkeit zieht herauf, eine Gemeinschaft derer, die sich als Brüder erachten, unsicher noch und tastend, aber doch zu allem entschlossen: Doch kann diese Gemeinschaft jenseits der Sklaverei der Arbeit gelingen?

In der letzten Strophe wendet sich das Ich nach dem ersten Vers zum zweiten Male an sein Herz, um sich sicher zu sein oder zumindest doch sich selbst zu versichern, dass diese Unbekannten alle Brüder sind. Die Kontinente sind zugrunde gegangen, die Vulkane explodieren und die Tiefen der Meere tun sich auf. Doch in der Liste fehlte einer: Afrika. Es sind die „unbekannten Schwarzen“, mit denen sich das lyrische Ich auf den Weg machen will, selbst wenn sich unter ihm die alte Erde öffnet und ihn in ihren Schoß zu ziehen sucht. 
Freilich: Das Ich hält - auch wenn sich unter ihm die alte Erde auftut - noch immer an dieser starken Vision fest, erblickt noch immer in den Schwarzen die Hoffnung, die es in der Alten Welt, aber auch in der Neuen nicht mehr finden kann. Es ist eine Anrufung Afrikas zu einem Zeitpunkt, als sich die dritte Phase beschleunigter Globalisierung zu entfalten beginnt, welche bald eine neue, globale Weltordnung und den Kampf der alten Mächte um die Hegemonie über weite Gebiete der Erde einläutet. Es fällt nicht schwer, darin die autobiographische Tatsache zu erkennen, dass Frankreich und Europa dem unsteten Rimbaud bald viel zu klein geworden waren und er nach Neuem, nach Unbekanntem strebte, das er aber nur außerhalb Europas finden konnte.

Selbst wenn wir Rimbauds Illuminations, Le Bateau ivre oder Une saison en enfer in unserer Analyse nicht berührt haben, so dürfte doch die ganze visionäre Kraft deutlich geworden sein, welche von diesem Jüngling ausging, dessen beredtes Verstummen noch immer über der modernen Lyrik wie eine dunkle Vorhersage schwebt. Er löste ein, was Baudelaire an absoluter Modernität forderte, und ging zugleich weit darüber hinaus auf anderen, neuen Wegen. Dies waren dichterische Wege, die tief in die historischen Avantgarden des 20. Jahrhunderts führen und noch den Neoavantgarden der zweiten Hälfte des zurückliegenden Jahrhunderts Bilder und Impulse lieferten. Mit der Anrufung der Schwarzen, der Brüder durchbricht das lyrische Ich von Qu'est-ce pour nous, mon coeur zugleich die Ordnung, jegliche Ordnung, selbst die koloniale Unterdrückungsordnung. In der dritten Phase beschleunigter Globalisierung, die in der dichterischen Schaffenszeit Rimbauds begann, entwickelte sich weltweit eine neue Ordnung, die erst mit dem Ende dieser Phase vor etwas mehr als einhundert Jahren zerbrach.

Die Aufteilung Afrikas unter die alten und neuen Kolonialmächte bei der Berliner Konferenz von 1884 sorgte dafür, dass die Rimbaud'sche Vision vom Weltenbrand sich nicht verwirklichte, sondern einer neuen, noch stärker ausplündernden Weltordnung Platz machte. Sie bildet den Hintergrund für die letzte Phase der Literaturen der Welt im 19. Jahrhundert, die wir nunmehr beginnen wollen. Doch die dem Franzosen und Europäer Arthur Rimbaud noch „unbekannten Schwarzen“ waren in den Literaturen der Welt, wie wir sahen, längst schon zu nicht mehr nur beschriebenen, sondern zu aktiven und fortan schreibenden Subjekten geworden. 